\title{
Critiques paratopiques. Ritter, Lambert et Jeanneret, des Neuchâtelois déracinés et leur rapport à la tradition
}

\author{
Dave LÜTHI \\ Université de Lausanne
}

\begin{abstract}
William Ritter, un homme exceptionnel ? Souvent isolé par l'historiographie en raison de son profil jugé atypique, Ritter n'est pas souvent remis dans son contexte historique et culturel. Cette contribution cherche à remédier à cette lacune en le comparant à deux acteurs contemporains de la critique artistique, deux autres Neuchâtelois, les architectes André Lambert et Charles-Édouard Jeanneret-Gris (bientôt plus connu sous le pseudonyme de Le Corbusier). Pratiquant comme Ritter une lecture paratopique de la scène artistique de leur temps, ils permettent de juger du degré d'originalité de leur confrère dilettante.
\end{abstract}

Keywords: critique d'art, voyage, paratopie, exotisme, patriotisme, Europe, Suisse

À lire la bibliographie encore peu fournie qui s'intéresse à William Ritter, il serait inclassable, décalé, unique en son genre mais, au contraire de son grand ami Charles-Édouard Jeanneret qui est souvent décrit de la même manière, Ritter est plutôt à l'arrière-garde qu'à l'avant. La personnalité complexe voire déroutante du personnage amène volontiers à ce type de constat, de même que pour son compère architecte. Dans les deux cas, le recours aux archives ne sont a priori pas d'une grande aide pour tempérer cette vision auctorielle, car les fonds documentaires ont été constitués par eux et par d'autres de manière à diriger le regard dans telle ou telle direction, donnant à voir la part jugée caractéristique des personnages - car c'est presque d'une mise en scène théâtrale dont il s'agit. En effet, comme Jeanneret, William Ritter a préparé son mémorial et dirige a posteriori les recherches le concernant. Ses fonds d'archives, classés, triés par Josef Tcherv, peu expurgés sans doute, imposent aux chercheurs une masse presque incommensurable de matériaux très divers - écrits, dessins, photographies - qui témoignent de son activité créatrice constante. Se plonger dans ses archives équivaut hélas souvent à une noyade, ce qui explique vraisemblablement encore la carence d'études concernant le critique d'art, auteur, musicologue, dessinateur, photographe amateur qu'il est. Par conséquent, les recherches portant sur le Neuchâtelois fonctionnent souvent en vase clos, confirmant l'intuition de départ : il est inclassable, décalé, unique.

$\mathrm{Ne}$ voulant en rien prétendre à une analyse fine du personnage, la présente contribution cherche à inverser cette perspective par le truchement 
d'une approche comparative de Ritter. En dégageant les lignes de force de sa pratique de critique d'art, en nous intéressant à son rôle de porte-parole paratopique $^{\mathrm{I}}$ - de $\mathrm{I} 888$ à I9I4, il parle presque toujours depuis ailleurs - on peut chercher à le situer dans le monde très internationalisé de l'avant Première Guerre mondiale. Deux autres Neuchâtelois, André Lambert, architecte et critique d'art, et Charles-Édouard Jeanneret, offrent des points de comparaison très utiles comme on le verra. Ritter et Jeanneret sont proches, on le sait; on ignore en revanche si Ritter et Lambert se connaissent - sans doute pas $^{2}$. Mais leurs parcours ne sont pas sans liens et la comparaison permet de cerner les éléments topiques ou exceptionnels de leur biographie respective, de même qu'avec Jeanneret.

\section{André Lambert et la double paratopie}

Né à La Chaux-de-Fonds 3 en I85I, André Lambert étudie l'architecture à l'École polytechnique de Stuttgart et à l'École des Beaux-Arts de Paris. Il travaille dans un premier temps à Neuchâtel dans l'atelier d'Eugène Colomb puis ouvre en I883 son propre bureau à Stuttgart avec son ami d'études Eduard Stahl (I849-I926), actif jusqu'à la Première Guerre mondiale. Il meurt en 1929 en Espagne, à Javea près de Valence, où son fils homonyme, illustrateur et graveur, avait fondé en 1920 un atelier de gravure ${ }^{4}$. De sa production architecturale, l'historiographie retient le Musée historique de Berne (I892-I894) mais c'est avant tout dans sa ville d'adoption, Stuttgart, qu'elle est effective ; là, il construit avec Stahl de très nombreuses villas et des immeubles de rapport (Breig 2000).

Ce qui nous intéressera ici, c'est moins son activité de constructeur que sa pratique de critique architectural. En effet, dès le début des années I890,

I Selon Dominique Maingueneau, la paratopie « peut prendre le visage de celui qui n'est pas à sa place là où il est, de celui qui va de place en place sans se fixer, de celui qui ne trouve pas de place. La paratopie écarte également d'un groupe (paratopie d'identité), d'un lieu (paratopie spatiale) ou d'un moment (paratopie temporelle). Distinctions au demeurant superficielles : comme l'indique le mot même, toute paratopie peut se ramener à un paradoxe d'ordre spatial. On y ajoutera les paratopies linguistiques (la langue que je parle n'est pas ma langue) » (http:// dominique.maingueneau.pagesperso-orange.fr/glossaire.html\#Para (consulté le I2 décembre 20I8)). Voir aussi Maingueneau (1993: 2I-33, 2004).

2 Le fonds conservé aux ALS ne contient aucune lettre de lui et la volumineuse correspondance entre Ritter et Jeanneret ne fait jamais référence à Lambert (Dumont : 20I4).

3 Les versions divergent entre les différentes sources, mais selon les registres d'immatriculation de la Technische Hochschule de Stuttgart et Suzanne Stelling-Michaud, Le livre du recteur de l'Université de Genève, IV, Genève, 1975 (Stelling-Michaud 1975 : 255, citant des sources aux Archives d'Etat de Neuchâtel), il est né à La Chaux-de-Fonds et non à Genève comme l'indiquent notamment Brun 1905 tome II : 217 et DHS.

4 André Lambert (1884-1967) est formé à Paris, ville qu'il aurait fui au profit de l'Espagne en raison du climat (Monter Pérez : 2013). 
il joue un rôle important dans l'édition d'architecture. D'une part, comme promoteur de ce qu'il appelle l'architecture « moderne », c'est-à-dire des édifices historicistes et éclectiques bien de son temps mais loin des courants les plus novateurs. En effet, au même moment émerge l'Art nouveau un peu partout en Europe : rien dans les édifices publiés par le Neuchâtelois dans son recueil au titre pourtant programmatique Moderne Architektur n'évoque de loin ou de près ce genre d'expérience en rupture avec l'ordre établi (Lambert \& Stahl I890). À l'inverse, on peut constater l'intérêt précoce de Lambert pour l'architecture régionaliste, cette tendance alors en plein essor en Allemagne, mais pas encore en Suisse, qui reprend des formes anciennes régionales pour créer une nouvelle architecture à la fois moderne car fondée sur un primat hygiéniste et rationnel, et traditionnelle car usant de formes et de matériaux censés être caractéristiques du lieu. L’un des édifices particulièrement intéressant de son recueil est une maison de style néo-renaissance édifiée à Neuchâtel en I887-I888 par les architectes Prince \& Colomb (ce dernier est l'ancien patron de Lambert) qui reproduit les formes très reconnaissables des façades locales du Xvi ${ }^{e}$ siècle - formes que Lambert semble apprécier puisqu'après les avoir publiées avec son collègue Alfred Rychner en 1883 déjà (Lambert \& Rychner I883), il les emploie au Musée de Berne, copiant la tourelle d'angle de la maison des Halles de Neuchâtel pour orner l'angle de son édifice. Son recueil de I883, L'architecture en Suisse aux différents époques, marque d'ailleurs un moment charnière dans l'historiographie du patrimoine bâti suisse : jusqu'alors, on s'était à peine intéressé à l'architecture traditionnelle de bois (en fait, un seul homme avait fait le travail, l'Allemand Ernst Gladbach, professeur de construction à l'École polytechnique de Zurich)'. Lambert et Rychner font un exercice similaire sur l'architecture de pierre dont ils cherchent à trouver la typicité helvétique, en mettant notamment en évidence le groupe particulièrement cohérent d'architecture renaissance neuchâteloise, celui-là même auquel se réfère stylistiquement l'immeuble de Prince \& Collomb.

Deux séries d'articles constituent le grand œuvre de Lambert. D’une part, il rédige vingt textes titrés « L'architecture contemporaine de la Suisse romande » qui sont publiés dans la revue alémanique Schweizerische Bauzeitung entre 1902 et 1908. D'autre part, quatorze articles intitulés "L'architecture moderne en Allemagne » et parus quant à eux entre 1905 et 1908 dans le Bulletin technique de la Suisse romande. Sans nous étendre trop ici sur le contenu, tirons-en quelques éléments pertinents pour notre propos. Il s'agit

5 Notamment: Der Schweizer Holzstil in seinen cantonalen und constructiven Verschiedenheiten vergleichend dargestellt mit Holzbauten Deutschlands, I868 puis sous une forme augmentée et en deux volumes en I882-I883 ; Die Holzarchitektur der Schweiz (1876) ; à titre posthume : Charakteristische Holzbauten der Schweiz, vom 16. bis 19. Jahrhundert, nebst deren inneren Ausstattung, Berlin, I898. 
de textes de présentation critique non seulement d'édifices et de bureaux d'architectes mais aussi de mouvements architecturaux que Lambert ordonne de manière raisonnée. C'est particulièrement vrai pour sa série «allemande " pour laquelle il construit une catégorisation très pertinente, à tel point qu'elle reste opérationnelle aujourd'hui encore pour qui s'intéresse à l'architecture allemande de la période wilhelminienne. Sa grande force est de partir d'un constat qu'il juge ojbectif et dont il fait la colonne vertébrale de son propos: celle d'une opposition fondamentale entre deux types d'inspiration contradictoire, la première ayant trait à l'histoire (historicisme ou refus de l'histoire au profit d'une modernité en rupture), la seconde à la géographie (inscription régionale ou langage international). Jouant avec les combinaisons ainsi possibles, Lambert décrit le monde qui l'entoure avec des catégories efficientes, allant du « modernisme reniant toute filiation avec le passé » à l'« art local avec reprise de la tradition historique ». Un jugement de valeur est sensible : il n'est guère favorable à la modernité lorsqu'elle est mal comprise, mais il loue les efforts de certains architectes, sis dans des villes considérées comme des centres artistiques ou des " écoles » (à l'instar de Karlsruhe, Dresde et Munich), pour renouveler des formes historicistes un peu répétitives. La recherche d'une architecture régionaliste, plus ou moins historiciste, semble toutefois plus intéressante à ses yeux car elle s'inscrit dans la tradition et joue la continuité plutôt que la rupture comme le pratique souvent les jeunes architectes - à la demande de leurs clients :

L’ensemble ancien [le village] était harmonieux parce qu'il avait été créé sous l'influence de traditions presque uniformes comme mode de construction et emploi de matériaux; chaque édifice et chaque maison, ayant bien son caractère particulier se soumettait cependant à une convention respectée et avait un air de famille. La dernière bâtisse vendue croit par contre se distinguer en méprisant la tradition et en montrant impudemment un type absolument étranger. L'harmonie est détruite, le village a perdu son aspect de bonhomie et de distinction ; la nouvelle bâtisse est déplacée et le paysage même souffre de ce désaccord (Lambert 1906:2).

Le discours est dans l'air du temps. Le Heimatschutz, association de sauvegarde du patrimoine bâti, se constitue dans les pays de langue germanique dans les années I89o alors que se développe le Heimatstil, le style régionaliste. Mais il n'est pas perçu comme rétrograde par tout le monde, car son refus, parfois, de l'histoire et de l'historicisme au profit d'un esprit, d'une ambiance, le délie d'un lien trop servile aux formes que l'historicisme puis l'éclectisme avaient successivement découvertes, employées puis modifiées durant tout le $\mathrm{XIX}^{\mathrm{e}}$ siècle. Dans son discours ambivalent, mais lucide et, il faut bien le dire, réaliste, Lambert se situe en marge des courants dominants, forcément dogmatiques - autant qu'un discours architectural peut 
l'être alors. Il fait part d'une hauteur de vue surprenante qui, à notre sens, doit beaucoup à son parcours personnel et professionnel.

En effet, l'architecte fait partie d'un petit groupe de constructeurs helvétiques expatriés, un phénomène assez mal connu encore. On peut mentionner Josef Anton Frölicher (I790-I866) qui reste en France comme architecte de la duchesse de Berry après ses études à Paris, sous le nom francisé de Joseph Antoine Froelicher (Macé de Lépinay 1976), ce qui ne l'empêche pas de construire dans la principauté alors prussienne de Neuchâtel (Collège latin et, peut-être, l'hôtel de Pourtalès-Castellane). Le Vaudois Jules Verrey (I822-I896) est un autre exemple intéressant. Établi un temps à Melun après ses études parisiennes, il rentre au pays dans les années I860. Du côté allemand, on peut citer le Zurichois Alfred Friedrich Bluntschli (I842-I930) qui ouvre en I87I un bureau florissant à Francfort avec son ami Jonas Mylius (I839-I883), né lui dans la principale ville de la Province de Hesse-Nassau ${ }^{6}$. Son activité allemande se ralentit avec sa nomination comme professeur à l'École polytechnique de Zurich en I88I. Enfin, citions encore Albert Frölich (I876-I953) et Otto Salvisberg (I882-I940) qui tous deux tentent, avec un succès variable, une double carrière allemande et suisse. La grande différence entre ces architectes expatriés et Lambert est la vision critique qu'ils ont de leur profession hors-sol. Si l'un ou l'autre rédige un article dans une revue pour présenter une réalisation, aucun de ces textes n'a l'ampleur ni l'ambition intellectuelle des séries d'articles de Lambert.

Le Neuchâtelois profite visiblement des conditions de sa carrière : établi (pour des raisons qui nous échappent) à Stuttgart, il y travaille et construit des édifices qui sont tout à fait dans l'air du temps, voire un peu dépassés pour certains - les immeubles et villas du bureau Lambert \& Stahl publiés dans Moderne Architektur ne témoignent pas, en tous les cas, d'une inventivité débordante. Mais sa prise de distance par rapport à la Suisse lui permet une lecture plus dégagée des débats internes de l'architecture de son pays d'origine, dont témoigne la série publiée en français dans l'organe germanophone de la Société suisse des ingénieurs et architectes. Sa maîtrise de l'allemand n'est-elle pas assez bonne pour qu'il puisse rédiger sans trop de problèmes ses longs textes dans sa seconde langue ? Cette hypothèse amène à penser que s'il est établi en Allemagne, il y reste toujours un étranger et que son intégration demeure relative. C'est peut-être justement grâce à cette distance linguistique et culturelle qu'il parvient produire, en français toujours, sa série sur l'architecture allemande, si objective (par rapport aux textes allemands contemporains) et clairvoyante. Cette double paratopie,

6 L'atelier remporte de nombreux concours prestigieux (hôpital de Heidelberg, cimetière de Vienne, hôtel de ville de Hambourg) et réalise d'importants édifices dans plusieurs villes allemandes (outre Francfort, les sources citent Heidelberg, Offenbach, Saarebruck, Mannheim, Constance, Worms) mais aussi suisse (Zurich) et italienne (Turin). 
qui le situe à distance de son pays d'origine par la géographie mais aussi de son pays d'accueil par une forme d'acculturation visiblement relative et critique, rend Lambert particulièrement intéressant et révélateur des courants de pensée de son temps. Il n'est pas anodin qu'il finisse ses jours en Espagne, dans un pays avec lequel il n'a a priori aucun rapport; cette rupture avec le «monde d'hier », comme l'intitule Stefan Zweig, est peut-être aussi un moyen pour Lambert de tourner la page après avoir pris conscience, justement, de la rupture définitive opérée par la guerre quant à la façon d'envisager le monde (architectural au moins).

\section{William Ritter}

Il n'est pas besoin de revenir ici sur les nombreux voyages effectués par le Neuchâtelois en Europe dès les années I890 et notamment dans les pays slaves (Dumont 2014 : I4-20 ; Donzé I999:6-8) ; bien plus mobile que Lambert, Ritter habite successivement à Paris, Prague, Bucarest et plus longuement à Vienne (I893-I898) et Munich (I90I-I9I4) (Kaenel I998 : 8I). En constant décalage - issu d'une famille fribourgeoise catholique, il grandit en terres réformées à Neuchâtel, est éduqué chez les Jésuites en France voisine puis au gymnase et à l'Académie de sa ville natale -, il rêve tout d'abord de Paris et de Bayreuth par le biais de ses passions pour les auteurs symbolistes et pour la musique de Wagner. Ritter découvre le Monténégro en I889 puis Bucarest en I890 à l'occasion d'un voyage initié par Léo Bachelin (I857-I930) son ancien professeur de grec à l'Académie, établi dès I889 en Roumanie et bibliothécaire du roi Carol ${ }^{\text {er7 }}$. Ritter se fond dans un monde qui lui correspond en négatif, si l'on peut dire : le dandy fin-de-siècle se complait ainsi dans un univers qu'il estime pur, authentique, traditionnel, riche d'une histoire dont le fil n'est ni rompu ni perverti. Durant ses voyages, il saisit de plusieurs manières ses impressions de voyage : dessins, photographies (Binggeli 2019) et descriptions écrites lui permettent de fixer un souvenir aussi vibrant que possible des contrées qu'il traverse. Toutefois, loin de se contenter de dessiner des architectures ou des paysages, il s'intéresse aussi aux costumes traditionnels, aux croix dans les cimetières, à tous les détails caractéristiques pour lui de la culture qu'il découvre. À l'instar de Lambert qui relève dans les années I880 les traits typiques de l'architecture suisse, Ritter fixe au début de la décennie suivante, mais pour lui-même, les contours formels de ce qu'il estime être le monde culturel slave. Hésitant entre le classique dessin de voyage et une perspective plus ethnographique,

7 Il avait en I882 déjà publié une étude sur les contes de fées roumains (Studii despre basmele românești). Sept contes seront publiés en version française en I894, introduits par lui (Sept contes roumains, Paris, 1894 ). 
il livre de manière de plus en plus subjective sa vision des nations qu'il traverse. En effet, les dessins des premiers voyages font place à des aquarelles, souvent de belle qualité, mais dont l'inscription topographique est plus floue, moins soutenue. Sans doute une fois l'excitation de la découverte passée, Ritter s'approprie-t-il formes et paysages au travers d'une technique picturale habile, qu'il développe peu à peu en lien avec ses goûts artistiques - en l'occurrence, un post-impressionnisme paradoxalement quelque peu hodlérien parfois. L'exotisme a fait place à l'habitude, mais une habitude toujours passionnée.

C'est dans ce contexte de déracinement perpétuel qu'il faut replacer sa production de critique d'art, si intense au début du $\mathrm{xx}^{\mathrm{e}}$ siècle notamment. Il collabore à plusieurs revues d'art et d'arts décoratifs de portée internationale comme la Gazette des Beaux-Arts, Art et Décoration, L'Art et les Artistes (Paris), Die graphische Kunst (Darmstadt), Emporium (Bergame) et L'Art moderne (Bruxelles) ${ }^{8}$. Sa contribution à L'Art et les Artistes dure de la fondation de la revue en 1905 jusqu'à la guerre de 1914 ; elle est particulièrement soutenue car Ritter y tient une chronique régulière dans la section « Mouvement artistique à l'étranger » et portant sur l'art à Prague puis, dès son emménagement à Munich, en Allemagne du Sud et en Autriche-Hongrie. On notera avec intérêt qu'il ne se charge pas de rendre compte de la production helvétique, la chronique étant tenue par plusieurs auteurs, souvent genevois, dont le peintre Charles Giron et le journaliste Gaspard Vallette, ancien rédacteur en chef de La Suisse. Les revues auxquelles participent Ritter comme auteur et comme critique font la promotion d'un art qualifié de moderne et qui correspond grosso modo à notre définition actuelle, floue et étendue, de l'Art nouveau. Rien de surprenant à cela puisque l'on connaît le goût de Ritter pour le symbolisme dans la droite ligne duquel se place cet Art en rupture avec le canon académique mais qui en conserve certaines des références, notamment celle à la nature, qui devient centrale dans les formes traitées par les revues en question. Les mouvements " géométriques ", à l'instar de la Sécession viennoise, ne trouve pas grâce à ses yeux, ni les peintres qui comme Hodler, tentent de raviver une soi-disant tradition suisse avec des formes caricaturales et des couleurs criardes, donnant un art " grossier et brutal » (Ritter I90I3 in La Semaine littéraire : 3I cité par Kaenel I998 : 92). C'est l'Art nouveau d'un Mehoffer ou le symbolisme de Böcklin qui sont les références absolues, entre autres artistes révérés par lui.

Si son activité de critique d'art permet tout d'abord à Ritter de vivre c'est son principal revenu -, il joue aussi un rôle fondamental dans la volonté qui l'anime de faire connaître l'art slave aux pays de l'Europe de l'Ouest.

8 Liste issue du manuscrit de Josef Tcherv « William Ritter, maturité I890-I9I4 » sur la vie de Ritter, version conservée à Neuchâtel : 298 (aimable communication de Xavier Galmiche). 
Nombre d'articles traitent en effet de l'art en Roumanie ; dans L'Art et les Artistes, ce pays n'a pas droit à sa rubrique mensuelle comme d'autres. Ainsi, c'est par le biais de véritables articles thématiques qu'il rapporte l'actualité de la production artistique roumaine aux lecteurs francophones. À titre d'exemple on peut citer sa visite de la collection royale de peinture ainsi que son compte rendu de l'Exposition nationale jubilaire de Bucarest, deux textes parus en I906-I907 (Ritter 1906a, I906b). Le premier décrit la collection constituée par Charles (ou Carol) I ${ }^{\text {er }}$ (I839-I9I4) au palais royal et qu'il a sans doute visité par l'entremise de Bachelin. Rappelons que le souverain est un membre de la famille de Hohenzollern élu prince régnant en I866 et proclamé roi en I88I. De la galerie de tableaux réunie, Ritter retient et illustre notamment un Ribera, un Cranach, un Clouet, deux Greco, un Rembrandt, des portraits de Fischbein, Reynolds et Greuze, témoignant d'un goût à la fois large et assez original. Ritter témoigne d'ailleurs d'une forme d'étonnement : «Et lorsque, sortant du palais, mes yeux encore pleins de la forte vision des chefs-d'œuvre espagnols qui sont la bonne aubaine d'une visite à cette Galerie, tombèrent sur la beauté fière et vigoureuse de la vie populaire roumaine éparse au plein soleil des rues, une étrange association d'idées se fit dans mon esprit » (Ritter I906-I907 : 278). Pour l'auteur, cette collection, comme le château tout entier, répond aux besoins identitaires du monarque contraint de quitter son Bade-Wurtemberg natal :

La Roumanie est un des plus beaux royaumes du monde, richesse, lumière, éclat, air de bonheur partout répandus. Il semble que nulle mélancolie n'y saurait atteindre. Et pourtant parmi cet or aveuglant et cette atmosphère ambrée une âme du Nord connaîtra de poignantes nostalgies, nostalgie surtout des douces pénombres et des ciels voilés. J'ai l'impression que le Souverain même qui a accompli sur cette terre merveilleuse de si grandes choses n'y a pas échappé, tant dans ses Demeures j'ai été frappé d'une sorte d'insistance avec laquelle [...] il s'est réservé des recoins d'ombre au milieu de boiseries austères et a fermé les fenêtres de ses appartements intimes de vitraux qui les isolent des aspects torrides de Bucarest. A Sinaia comme au Palais Royal il a eu besoin d'un décor Renaissance allemande qui lui donnât l'illusion des châteaux de son pays. Il y a appelé un vieillard de légende, le maître huchier de son auguste père ; et le mobilier de Castel Pelesch a été voulu semblable à celui de Sigmaringen [son lieu de naissance] (Ritter I906-I907 : 272).

De l'Exposition Nationale jubilaire de Bucarest, Ritter ne retient guère que la salle de son peintre ultra-favori, Nicolae Grigorescu, sur lequel nous reviendrons. Sinon, « la grande beauté de cette exposition, la voici. Elle démontre l'existence d'une architecture nationale » qui 
se témoigne apte à imprimer un caractère décisif aux aspects d'une capitale jusqu'ici sans silhouette, comme sans parti pris; elle se prouve capable de répondre à toutes les vastes exigences de la vie moderne qui veut de l'espace, de l'air, ses aises et ses orgueils. L'hôtel des postes de Bucarest, la Caisse des Dépôts et Consignations, l'Athénée, le Palais Royal et les demeures des boyards et des nababs roumains ne seraient plus possibles aujourd'hui dans un style autre. Et je me demande même si Castel Pelesch (Sinaïa), nette affirmation de l'origine allemande d'un souverain à cette heure complètement roumanisé, n'obéirait pas, entrepris aujourd'hui, à cet idéal nouveau (Ritter 1906a: V).

Une digression sur les motifs d'architecture nationale, ceux-là même que Ritter dessinait quinze ans plus tôt dans ses carnets, lui permet de louer certains édifices de l'exposition, dont le pavillon royal et une chapelle qui copie l'église Saint-Nicolas de Iași.

Comme Lambert, Ritter fait la promotion de l'architecture régionaliste par le biais de son texte. La portée de son propos est a priori tout autre. Alors que l'architecte donne une vision critique et raisonnée de l'utilisation des formes traditionnelles qui ne doivent pas enfermer la création contemporaine, Ritter en vante lui les vertus idéologiques. Mais tous deux se rejoignent sur l'essentiel : la tradition peut être le socle de la modernité ; revisitée pour le premier, plus servile pour le second - ou, si l'on préfère, plus historique, pour reprendre l'un des concepts-clé de Lambert.

Le même type de réflexion guide les propos de Ritter concernant l'œuvre de Nicolae Grigorescu mais avec un sujet différent ${ }^{9}$. En effet, si la peinture de ce peintre majeur en son temps en Roumanie traite de thèmes "régionaux » (Ritter publie, par exemple, un Déménagement de tziganes et un Jeune tzigane), d'autres sont moins connotés (Les Bœufs, La Bergère). Quant au style du peintre, tout brumeux de sa touche large, souvent empâtée et sombre, il n'a pas grand chose de local puisque la formation de Grigorescu se déroule dès I86I à l'École des Beaux-Arts de Paris, chez Cornu, en même temps que Renoir. Il a des contacts avec des membres de l'École de Barbizon (Millet et Corot pour ne citer que les plus connus) et par le biais de Georges de Bellio, il découvre les impressionnistes. Un voyage en Italie, en Grèce, à Vienne et dans les Balkans voit son goût s'affirmer mais où qu'il soit, c'est le monde paysan qui l'intéresse - preuve s'il en faut que le « motif » n'a rien de roumain, quoi qu'en pense Ritter. Ce n'est qu'après un long séjour à Paris et en Bretagne entre 1879 et 1890 qu'il rentre au pays ; il expose régulièrement à l'Athénée et devient membre honoraire de l'Académie roumaine. À sa mort en 1907 , il passe pour le fondateur d'une école moderne de peinture.

9 Outre l'article de Ritter (1906a), voir Enache (2012), Nicolae Grigorescu (2006) ; Macovei (1999). 
Ritter est quelque peu aveuglé par sa passion pour le peintre, au contraire de l'objectif froid de Lambert, rarement enthousiaste. Ainsi, de l'œuvre du Roumain, Ritter dit qu'elle

est si libre, si aisée, si exempte de prétention, d'une composition si spontanée et empirique, d'une belle matière simple et propre si dépourvue d'artifice, si indifférente à toute recette, que la classification n'en saurait même être chronologique. Si je la devais tenter, il la faudrait établir par la géographie, ou par les saisons, ou par le folklore. On n'a pas même l'idée de l'analyser en un essai soigneusement ordonné et de proportions savantes (Ritter 1906c : I66-I67).

C'est à peu près ce que disent certains contemporains, membres du Heimatschutz ou proches du mouvement Arts E Crafts en Angleterre, de l'architecture néo-traditionnelle, qui semble avoir toujours existé et ne dépendre d'aucune loi académique : le topos résulte bien d'une vision de l'esprit du temps, d'un Zeitgeist, témoignant de l'assimilation profonde d'une forme de folklore par la culture savante, réduit à ses caractéristiques formelles et décoratives censées transmettre des valeurs morales, patriotiques, voire politiques et religieuses. La question de l'occidentalisation de la culture slave inquiète beaucoup Ritter, qui tente par conséquent d'orientaliser la culture occidentale par le biais des œuvres et de la culture qu'il donne à voir et à connaître.

\section{Charles-Édouard Jeanneret}

Grâce notamment à la correspondance entre Ritter et Jeanneret publiée par Marie-Jeanne Dumont (20I4), on sait maintenant ce que ce dernier doit à Ritter pour la préparation de son célèbre voyage d'Orient, dont il a luimême entretenu et valorisé le souvenir par la publication tardive de ses dessins et notes, soigneusement triés ${ }^{\mathrm{I}}$. Comme Ritter, et comme Lambert dans un certain sens, il prend note de son voyage par le crayon - il écrit, il photographie mais, surtout, il dessine. Son intérêt le porte avant tout à observer l'architecture, quelle qu'elle soit, mais il ne néglige pas non plus les paysages, particulièrement en Bulgarie. On retrouve chez Jeanneret ce trait ample et souple, rapide aussi, pratiqué par Ritter dans les années I890, qui témoigne d'un dessin pris sur le vif, rapidement traité, mais d'un point de vue stylistique, qui doit beaucoup à son temps - on est parfois assez près de Van Gogh" plutôt que du dessin des architectes académiques contempo-

Io Le Corbusier, Le Voyage d'Orient, Paris, 1966. À ce sujet, voir notamment Gresleri (1984 : 26-36) surtout, et L'invention d'un architecte... 2013.

II Voir par exemple le dessin de la campagne bulgare publié par Gresleri (1984 : I55). 
rains qui négligent le trait au profit de la couleur. Dans les Balkans, il semble succomber comme Ritter à un certain goût foklorique; ses photographies de maisons dans les villages entre Baja et Belgrade montrent tant des constructions traditionnelles que des scènes villageoises où l'on retrouve les thèmes chers à Ritter et à Grigorescu : paysans sur un char ou dans un verger, berger faisant boire des ânes, cimetière aux croix de pierres incisées, et habitants pris de dos, chargés d'habits et de sacs... Difficile de penser que Jeanneret ne fait que reproduire des images qu'il aura pu voir chez son mentor; pourtant, il faut bien reconnaître que pour le reste de son voyage, ailleurs que dans les Balkans, ce type de dessins et de photographies est plus rare. Il se concentre en effet avant tout sur l'architecture, occidentale ou orientale et généralement sans architecte et sans âge, des maisons en briques des villes anciennes allemandes aux maisons en bois posées sur les rives du Bosphore à Istanboul.

L'usage que fera Jeanneret de ce matériau diffère complètement de celui de Lambert et Ritter. Le premier utilise les formes traditionnelles suisses qu'il étudie pour ses propres réalisations, comme autant de citations donnant une âme et des racines à son architecture, quand ce n'est pas celles des autres qu'il diffuse par leur publication. Ritter ne cesse quant à lui de chanter les louanges d'un monde préservé, authentique, qui ne peut être qu'admiré. Grigorescu devient pour lui le porte-étendard d'un monde en marge, en danger, mais qui devrait être un modèle. Jeanneret, reprendra des idées issues de ses voyages mais de manière très remaniée ou retranscrites. Ainsi, pour la villa Schwob à La Chaux-de-Fonds, construite peu après son retour et rapidement qualifiée de Villa Turque, grandes surfaces lisses de mur, toiture plate, fenêtres fermées par des sortes de claustra évoquant des moucharabieh sont autant de traces de ce voyage. Mais il faut sans doute être prudent avec la manipulation des potentiels motifs ; ainsi, son projet pour les Ateliers d'art à La Chaux-de-Fonds (I9IO) est souvent rattaché typologiquement aux mosquées qu'il visite dans les Balkans ainsi qu'à la chartreuse de Ema. Pourtant, ce n'est qu'en I9II qu'il découvre ces monuments... Quoiqu'il en soit, Jeanneret détourne complètement et systématiquement ce qu'il a vu, à tel point qu'il est presque impossible de déceler tel trait « folklorique » digéré par lui. Sa modernité à lui est bien fondée sur une étude d'une architecture ancienne et régionale, mais cette fois-ci, c'est moins les formes qui comptent que les principes.

\section{Local / global}

Une rupture s'opère, celle d'un monde dont les références vont complètement basculer notamment en raison des changements paradigmatiques impliqués par la Grande Guerre. La globalisation du conflit implique celle 
du commerce et de l'économie, puis de la culture. Le passé, forcément inscrit dans un terreau local, régional ou national, est le grand perdant de ce changement idéologique. Jeanneret, devenu Le Corbusier, en fera sa force. Ailleurs, à Javea et à Melide, Lambert et Ritter attendront tous deux leur heure : leur monde a disparu et ils n'ont pas les capacités culturelles de réagir à un changement aussi brusque. Ritter raillait quelque peu le roi des Roumains de se recréer un monde germanique pour faire disparaître la nostalgie de ses origines ; son exil au bord d'un lac alpin n'est peut-être pas sans nostalgie non plus, comme un rappel à la fois du lac tant aimé de son enfance, à Neuchâtel, et de ceux de Munich qu'il a si souvent dessinés, ainsi que des paysages montagneux des Balkans qui ont contribué à la construction si particulière de son image du monde.

À chacun sa vision paratopique : celle de Ritter lui a permis à la fois de découvrir un monde et de le faire découvrir. Les effets de sa production publiée sont sans doute sans commune mesure avec celle de Lambert, qui anticipe un mouvement architectural d'extrême importance en Suisse durant vingt ans, ni avec celle de Le Corbusier qui saura faire fructifier l'expérience de ses voyages et de son exil en France durant toute sa carrière. Mais à leur manière, tous trois montrent comment, dans le monde d'avant la Première Guerre, le texte programmatique est un véritable outil pour les intellectuels, en particulier pour ceux qui, comme ces trois Neuchâtelois, vivent en partie dans un exil plus moins volontaire. Se faire entendre, exister sur une scène artistique en pleine mutation et, surtout, en pleine institutionnalisation : dans un monde aussi petit que la Suisse, la prise de distance permet d'autant mieux une forme de distance. Si Ritter se fond dans une critique parfois acerbe, comme celle de Jeanneret, Lambert montre plus de recul par rapport aux conditions de création de son pays d'origine. Mais le résultat importe finalement peu : le constat de l'urgence de l'expression, à une époque de grande vitalité culturelle, est sans doute ce que cette comparaison permet de vérifier le mieux. 


\section{Bibliographie}

Binggeli, Thomas, «William Ritter photograph(i)e », Versants, 66:I, 20I9, pp. 97-I23.

Breig, Christine, Der Villen- und Landhausbau in Stuttgart I830-I930 : ein Überblick über die unterschiedlichen Umsetzungen und Veränderungen des Bautypus Villa in Stuttgart, Stuttgart, Hohenheim, 2000.

Brun, Carl, Schweizerisches Künstler-Lexikon, 4 vol., Frauenfeld, Huber, I9041905.

Donzé, Fernand, Calame, Caroline \& Charrière, Edmond, William Ritter (I867-I955) au temps d'une autre Europe : dictionnaire à l'usage des curieux de la vie et de l'oeuvre d'un Neuchâtelois hors du commun, Neuchâtel, Nouvelle Revue neuchâteloise, $\mathrm{n}^{\circ}$ 6I, 1999.

Enache, Monica, Nicolae Grigorescu (I838-I907) : l'âge de l'impressionnisme en Roumanie, Milan, Silvana, 2012.

Dumont, Marie-Jeanne (éd.), Le Corbusier. Lettres à ses maîtres. Le Corbusier, William Ritter, correspondance croisée I9IO-I955, Paris, Linteau, 2014.

Gladbach, Ernst, Charakteristische Holzbauten der Schweiz, vom I6. bis I9. Jahrhundert, nebst deren inneren Ausstattung, Berlin, Claesen, I870.

- Der Schweizer Holzstil in seinen cantonalen und constructiven Verschiedenheiten vergleichend dargestellt mit Holzbauten Deutschlands, Zurich, Schmidt, I868.

-. Die Holzarchitektur der Schweiz, Zurich, Füssli, I876.

Gresleri, Giuliano, Viaggio in Oriente : gli inediti di Charles-Édouard Jeanneret fotografo e scrittore, Venise, Marsilio, I984.

Jeanneret-Gris, Charles-Édouard, dit Le Corbusier, Le Voyage d'Orient, Paris, Les Forces Vives, 1966.

Kaenel, Philippe, "William Ritter (I867-I955) : un critique cosmopolite, böcklinien et anti-hodlérien ", Revue suisse d'histoire, 48, I998, pp. 73-98.

L'invention d'un architecte : le voyage en Orient de Le Corbusier, Paris, Villette, 2013.

Lambert, André \& Rychner, Alfred, L'architecture en Suisse aux différentes époques, Bâle, Genève, Georg, I883.

Lambert, André \& Stahl, Eduard, Moderne Architektur. Ausgeführte städt. Wohngebäude, Geschäfts- $u$. Einfamilienhäuser, Villen mit ihren Nebenbauten, interessante Details u. Innenansichten, Holz- $u$. Zierbauten, Grundrisse etc. in farb. Darst., Stuttgart, Wittwer, I890.

Macé de Lépinay, François, «De Soleure au Faubourg Saint-Germain : Joseph-Antoine Froelicher (I790,I866), architecte de la duchesse de Berry », Revue suisse d'art et d'archéologie, 3, 1976, pp. 211-223.

Macovei, Catalina, Nicolae Grigorescu, Bournemouth, Parkstone, 1999.

Maingueneau, Dominique, Le contexte de l'œuvre littéraire, Paris, Dunod, 1993. 
—. Le Discours littéraire. Paratopie et scène d'énonciation, Paris, Armand Colin, 2004.

Nicolae Grigorescu (I838-1907). Itinéraires d'un peintre roumain de l'école de Barbizon à l'Impressionnisme, Paris, Somogy, 2006.

Monter Pérez, Jose, Los Lambert entre París y Xàbia, Valence, Museu de la Il-lustració i de la Modernitat, 2013.

Ritter, William, "L'art à l'Exposition nationale jubilaire de Bucarest », L'Art et les Artistes, I906a, I9, supplément illustré, pp. IV-IX.

—. "La Galerie de Tableaux du roi Charles I ${ }^{\text {er }}$ de Roumanie ", L'Art et les Artistes, 1906b, 19, pp. 272-279.

—. « Nikoulae Ion Grigoresco », L’Art et les Artistes, 1906c, II, pp. I66-I67.

—. «La Suisse à l'exposition de Munich », dans La Semaine littéraire, 28 juin I9I3.

-.Études d'art étranger, Paris, Mercure de France, 1906d.

Stelling-Michaud, Suzanne, Le Livre du recteur de l'Université de Genève, IV, Genève, Droz, 1975. 